\title{
Erratum: Polariton Dispersion of Periodic Quantum Well Structures (Pis'ma Zh. Éksp. Teor. Fiz. 76, 739 (2002) [JETP Lett. 76, 637 (2002)]
}

A. V. Mintsev, L. V. Butov, C. Ell, S. Mosor, G. Khitrova, and H. M. Gibbs

PACS numbers: 73.21.Fg; 71.36.+c; 73.20.Mf

In the article of A.V. Mintsev, L.V. Butov, C. Ell, S. Mosor, G. Khitrova, and H.M. Gibbs published in vol. 76, no. 10, pp. 637-640, Figures 1 and 2 should be reversed. 